Mediterránea Ser. Biol. (1985), n. ${ }^{\circ}$ 8. Págs. $59-72$

\title{
INFLUENCIA SOBRE LA DIVERSIDAD DE LOS VALORES DE IMPORTANCIA CONSIDERADOS EN EL MUESTREO
}

\author{
por \\ A. Puerto Martín 1 y J. M. Gómez Gutiérrez1
}

\section{RESUMEN}

Se analizan las variaciones que experimenta la diversidad al considerar seis valores de importancia diferentes: densidad, cobertura, biomasa, abundancia en función de la densidad, abundancia en función de la cobertura y frecuencia. El ejemplo que se propone incluye treinta comunidades agrupadas en seis tipos; tres de estos tipos son comunidades típicas de especies herbáceas del oeste semiárido español y los otros tres presentan distînta intensidad de especies leñosas.

Se concluye que las abundancias y la frecuencia son poco válidas para el cálculo de la diversidad. Densidad, cobertura y biomasa forman una serie continua que, en el orden en que han sido mencionadas, presentan un mayor rango de variación entre valores extremos (aspecto positivo) y una fuerte disminución de la influencia de la riqueza frente a la equitabilidad (aspecto negativo). Debido a esta antítesis de calidad se opta por la cobertura como mejor difinidora de la diversidad.

\section{SUMMARY}

An analysis is made of the variatons undergone by diversity on considering six variables of different importance: density, cover, biomass, abundance as a function of density, abundance as a function od cover and frequency. The example proposed includes thirty communities grouped into six types; three of these were typical communities of herbaceous species of the semiarid regions of Western Spain and the other three show different intensities of lignous species.

It is concluded that the abundances and frequency are of little interest for the calculation of diversity. Density, cover and biomass form a continuous series which, in the order mentioned, display a greater range of variation between extreme values (positive aspect) and a strong decrease of the influence of richness versus equitability (negative aspect). Owing to this antithesis in quality, cover is chosen as the best parameter or defining diversity.

(1) Departamento de Ecología. Facultad de Biología. Universidad de Salamanca. 


\section{INTRODUCCION}

Un aspecto que se presta a la síntesis en las çomunidades naturales es la evaluación de su estructura, por lo que nada tiene de extraño que se hayan intentado distintos procedimientos para resumir en una o pocas cifras la forma en que se disponen y combinan las diferentes especies. El pensamiento de que debían existir determinadas regularidades en la distribución de los individuos en especies es muy antiguo, y pone bien de manifiesto esta tendencia a la simplificación como un medio de hacer accesible la complejidad natural. Dicha tendencia se ha mantenido durante toda la historia de la Ecología, llevando implícita a veces una cierta candidez, pero de todas formas ha contribuido a que se examinen reiteradamente problemas de interés básico.

Por otra parte, los aspectos estructurales, contemplados desde un punto de vista práctico y directo, radican en que las probabilidades de aparición tanto de individuos como de especies no son iguales en distintas áreas o zonas. Considerando estas áreas por separado, pueden observarse en ellas peculiaridades de jërarquización, en el sentido de que determinadas especies, más o menos numerosas, destacan por sus valores de importancia, mientras que otras admiten el calificativo de subordinadas.

Así, han ido surgiendo numerosas expresiones que tratan de reflejar la diversidad de las comunidades, la mayoría de las veces a través de taxocenosis concretas. En realidad, un índice de este tipo es de formulación relativamente sencilla, ya que, como indica MARGALEF (1974), puede emplearse cualquier función monótona que alcance un mínimo cuando todos los elementos pertenezcan a la misma clase y un máximo cuando todos los elementos pertenezcan a clases distintas. Otra cosa es que dicho índice presente unas características adecuadas para la resolución de problemas concretos, lo que deja en entredicho la apreciación de PEET (1975) acerca de que las cualidades particulares que puedan tener cada uno de los índices no requieren ser destacadas, sino más bien contempladas con indiferencia. En efecto, no todos los índices son idóneos para la totalidad de los casos. Un índice de diversidad correcto debería ser poco sensible al tamaño de la muestra, ya que de estar muy influenciado por él desvirtuaría los resultados cuando estos quieran proyectarse a la comunidad objeto de estudio. A la vez, ha de ser invariante ante operaciones de selección realizadas en las muestras, como el uso de determinadas técnicas de muestreo o la elección de cierto grupo taxonómico. 
La consecuencia es que la proposición de un nuevo índice suele ir acompañada, aparte de por las bases matemáticas que presumiblemente lo hacen fiable, de comparaciones con otros ya existentes (MacINTOSH, 1967). Además, existen varios trabajos donde se ponen de relieve sus ventajas o desventajas, bien desde un plano preferentemente teórico (PIELOU, 1975) o centrándose en aspectos aplicados (GARCIA DE VICUÑA, 1982).

No es nuestro propósito volver a incidir en este aspecto, sino que a partir de una expresión considerada como adecuada, se utilizarán distintos parámetros de los usados comúnmente en la cuantificación de la vegetación. Conocer cual de estos parámetros presenta una sensibilidad mayor frente a los cambios estructurales y una adecuación más efectiva en casos concretos es importante, ya que ayudará a comprender mejor como queda mediatizada la diversidad y sus variaciones por los datos empleados, permitiendo comparar los resultados de trabajos en que se recurre a medidas distintas.

Por tanto, el objetivo que se plantea es práctico y metodológico. Conviene destacar que la diversidad se presta a una gran posibilidad de ramificaciones y, conceptualmente, la teoría que soporta en cuanto a sus relaciones con la estabilidad, implicaciones con situaciones futuras, captación de información ambiental, etc., es una de las más complejas dentro de la temática ecológica. Sin embargo, las apreciaciones prácticas quedan muy atrás del proceso intelectual que le ha concedido esta posición privilegiada; la diversidad se presenta aquí con un carácter preferentemente operativo, en la búsqueda de regularidades que quizá sirvan para establecer precisiones sobre conceptos actualmẹte vigentes.

\section{MATERIAL Y METODOS}

El estudio se ha realizado en treinta comunidades, incluibles a priori en seis grupos fisonómicos constituidos por cinco comunidades cada uno. Tres de estos grupos son representantes de los clásicos sectores del sistema vaguada, unidad repetitiva y paisajística de las superficies semiáridas españolas, que para la provincia de Salamanca (área de muestreo) ha sido descrita en varias ocasiones (GOMEZ GUITIERREZ et al., 1978; LUIS y MONTSERRAT, 1979; PUERTO et al., 1983). Así, el de pastizales húmedos corresponde a la zona de depósito, el de vallicares a la de transporte, y el de pastizales de efímeras a la de erosión. Los otros tres grupos, todos ellos de posición topográfica elevada, tienen como componentes comunidades invadidas por leñosas, de manera que se sigue la secuencia: matorral con amplios claros, matorral con claros pequeños y matorral denso. 
Las especies herbáceas de cada una de las comunidades fueron muestread̦as por medio de diez unidades elementales cuadradas de $0.5 \times 0.5 \mathrm{~m}$. Para las leñosas se establecieron cuadrados de $3 \times 3 \mathrm{~m}$, también con diez repeticiones, lo que pareció suficiente en función de su porte. Los parámetros de cuantificación comprenden seis aspectos: densidad (efectuando el recuento del número de individuos), abundancia en función de la densidad (según la escala de cinco grados recogida en CLAVER, 1982), cobertura (tanto por ciento de terreno cubierto), abundancia en función de la cobertura (escala de cinco grados; ver MARGALEF, 1974), biomasa (dada como materia seca por unidad de superficie) y frecuencia (número de ưnidades - máximo de diez- en las que cada especie estaba presente).

Como es fácil de observar, se incluyen tres caracteres discretos y de evaluación muy limitada (abundancia según la densidad, abundancia según la cobertura y frecuencia) junto con otros tres continuos y de valoraciones mucho más amplias (densidad, cobertura y biomasa). Las anotaciones no presentaron grandes dificultades, dependiendo éstas más bien del gran número de unidades muestreadas, excepción hecha de la biomasa, que requirió de la ayuda de un equipo bastante numeroso. $\mathrm{Su}$ cálculo se efectuó mediante corte en el campo de cada especie por separado, obteniéndose muestras que posteriormente fueron desecadas en estufa de aire forzado durante 24 horas para las herbáceas y 36 horas para las leñosas; dichas horas corresponden a los tiempos que, en las pruebas previas, habían sido calculados por exceso para llegar a peso constante.

Para el cálculo de la diversidad se emplea la conocida expresión (H') de SHANNON-WEAVER (1963), aplicada con logaritmos de base 2. Dicha expresión, según indica ODUM (1972), recoge bien la acción combinada de las dos componentes de la diversidad (riqueza y equitabilidad), por lo que cabe esperar de ella una respuesta de tipo intermedio; el saber hasta qué punto se encuentra centrada esta respuesta, es otro de los objetivos propuestos.

Las cualidades de este índice hacen que sobresalga como el más adecuado para el tipo de estudio que nos proponemos (GARCIA DE VICUÑA, 1982), por lo que nada tiene de extraño que su aceptación haya sido muy amplia (MONK, 1967; LOUCKS, 1970; AUCLAIR and GOFF, 1971; SHAFY and YARRANTON, 1973; NICHOLSON and MONK, 1974; WILLSON, 1974; BAZZAZ, 1975; TRAMER, 1975; WHITTAKER and NIERING, 1975; RICO, 1981; STERLING et al., 1984; etc.). No obstante, en el momento de realizar una elección de este tipo, siempre permanece un cierto subjetivismo, ya que como señala PUERTO 
(1977), no se puede olvidar que se está tratando de un concepto ecológico que deja abiertas las puertas de la ambigüedad a su interpretación matemática.

En cuanto a las dos componentes de la diversidad, como medida de la riqueza se empleará sencillamente el número de especies (S), y para la equitabilidad el cociente entre la diversidad obtenida y la máxima posible contando con ese número de especies ( $\left.\mathrm{J}^{\prime}\right)$.

\section{RESULTADOS Y DISCUSION}

En la tabla I se recogen los valores medios y las desviaciones típicas de diversidad ( $\left.\mathrm{H}^{\prime}\right)$, equitabilidad ( $\mathrm{J}$ ') y riqueza $(\mathrm{S})$, correspondientes a cada uno de los seis grupos de comunidades mencionados y a los distintos parámetros considerados. Dado que las desviaciones típicas para cada grupo y parámetro son en todos los casos muy pequeñas, se ha preferido trabajar con las medias, ya que éstas ofrecen los mismos resultados que los valores individuales pero permiten una simplificación mayor.
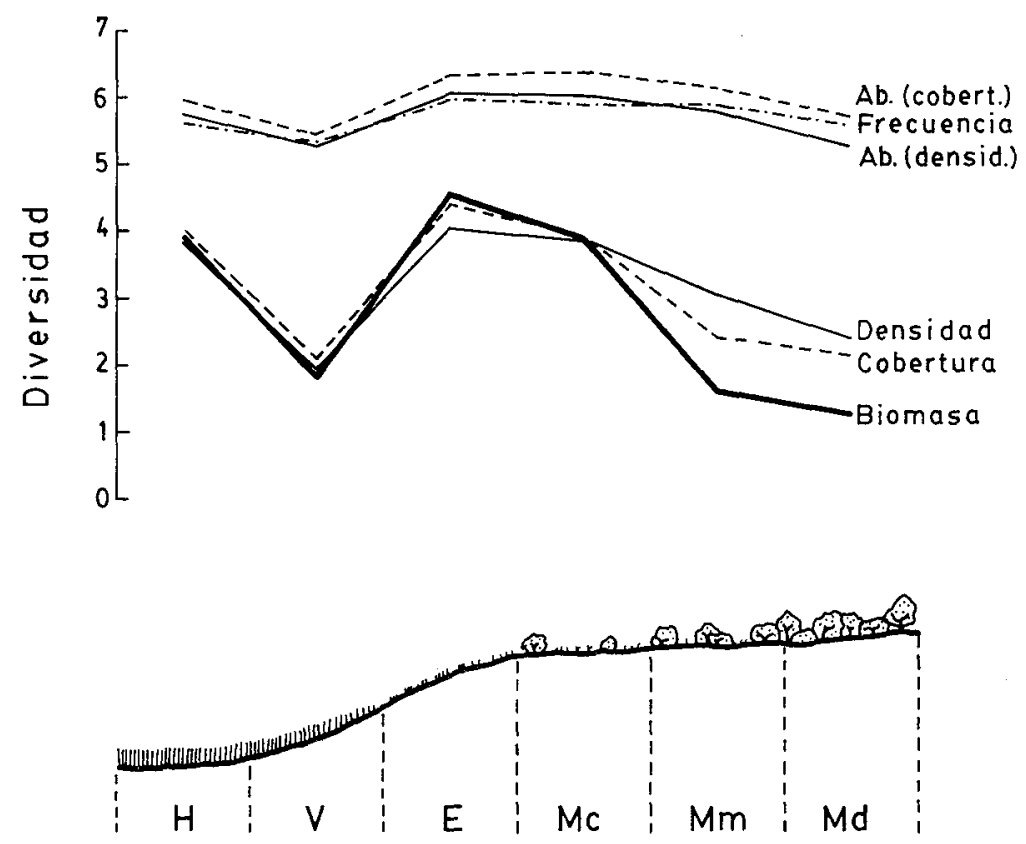

Fig. 1.-Evolución de la diversidad media, para los seis parámetros considerados, siguiendo un esquema idealizado en la disposición sobre el terreno de las distintas comunidades tipo. 


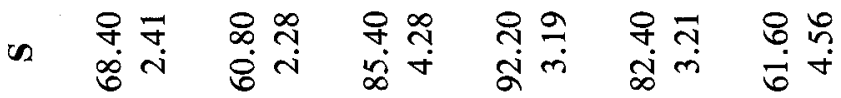

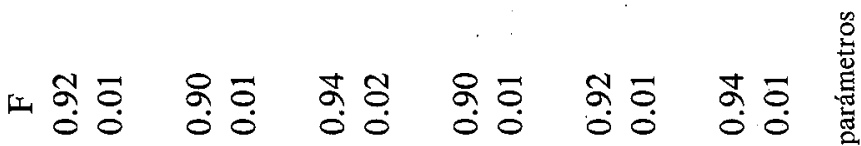

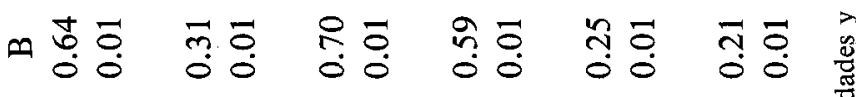

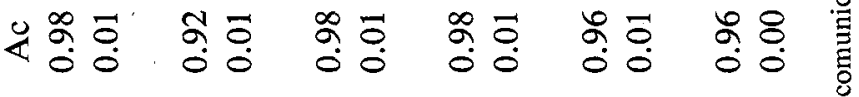

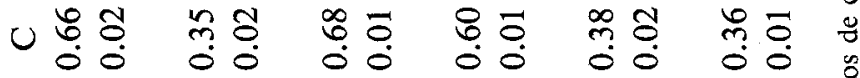

४⿳厶大

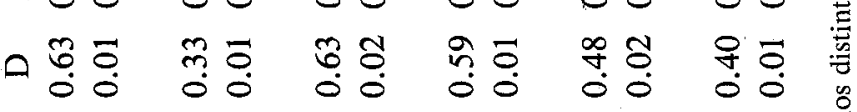

I

๓

«

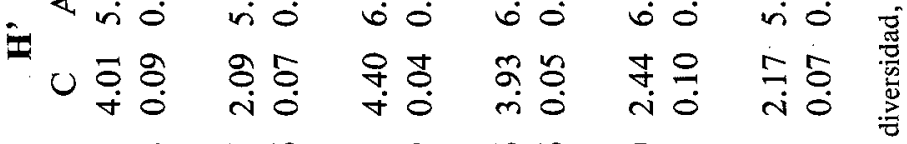

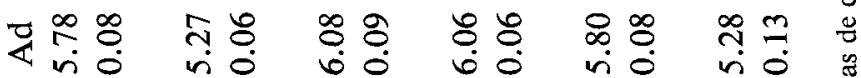

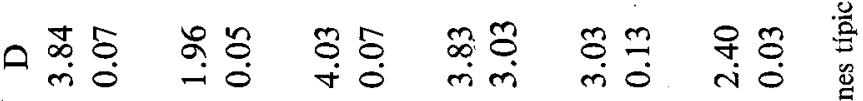

$x$ in $x$ in $x$ in $x$ in $x$ in $x$ in

吾

$\stackrel{0}{0}$

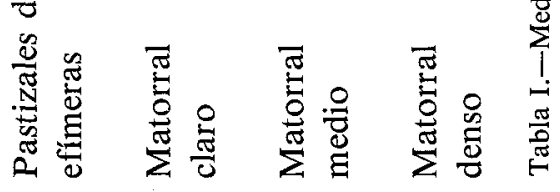


Así, la figura 1, muestra una seriación de las comunidades tal como puede deducirse de su posición habitual sobre el terreno, aunque las tres fases de matorral rara vez han sido muestreadas de forma continua. El esquema se basa en la noción de vaguadas «idealizadas», recogida por PUERTO et al. (1983), según la cual los análisis de ordenación efectuados con parcelas esparcidas en el espacio proporcionan gradientes tróficos similares a los que se obtienen en el estudio estratificado de vaguadas. Esto permite una cierta concisión, que hace más sencilla la exposición de los resultados.

En la parte superior de la figura se dispone la evolución de la diversidad, para las seis medidas básicas efectuadas, siguiendo el gradiente conceptual establecido. Como cabía esperar, se forman dos grupos; el primero de ellos, con valores de diversidad más elevados, corresponde a los parámetros que presentan un límite cuantitativo bajo en función de la gradación atribuida (caso de las abundancias) o del número de unidades elementales de muestreo empleadas (frecuencia). El segundo grupo está constituido por los tres parámetros restantes, que presentan un límite cuantitativo elevado (cobertura; máximo para una especie de 100\%) o dicho límite resulta en principio indefinido (densidad y biomasa).

Con todo, en una apreciación general, cabe reseñar que se presenta una misma tendencia en las variaciones. De una diversidad relativamente elevada en los pastizales húmedos $(\mathrm{H})$, donde son poco manifiestos los fenómenos de dominancia, posiblemente debido a que la acentuada presión del ganado queda compensada por la abundancia en agua y nutrientes, se pasa a cifras bajas en los vallicares de media ladera (V) que, a la característica de utilización intensa, unen condiciones tróficas sólo de tipo medio. En los pastizales de efímeras (E) los valores se incrementan de nuevo (se alcanza el máximo para cinco de los seis parámetros considerados, siendo excepción la abundancia en función de la cobertura), ya que su escasa calidad reduce el pastoreo en las explotaciones extensivas, estando mucho más frecuentados por los herbívoros los enclaves más eutrofos de posiciones medias o bajas; a ello se une el hecho de que son muchas las especies adaptadas a estos suelos pobres, donde siempre encuentran la posibilidad de implantarse, sin llegar a dominar, debido a las bajas coberturas existentes. En la zona de matorral con amplios claros (Mc), la diversidad sigue siendo elevada, si bien decae algo, excepto cuando la valoración se efectúa por la abundancia en función de la cobertura, experimentando entonces un pequeño incremento (aquí se sitúan las cifras máximas para este parámetro). A continuación se produce un descenso, cuando el matorral alcanza proporciones medias (Mm), que continúa a medida que las leñosas se hacen más densas $(\mathrm{Md})$, tanto debido a su propia presencia, lo que afecta 
sobre todo a cobertura y biomasa, como por razones derivadas de su efecto, que impone una selección en favor de determinadas especies herbáceas, lo que incide decisivamente en los otros cuatro valores de importancia considerados.

Si bien la evolución es paralela, es obvio que no ocurre así con los rangos de variación. La diferencia entre el máximo y el mínimo de diversidad (este último se encuentra siempre en los vallicares, excepto para la biomasa, en que es detentado por el matorral más denso) es muy pequeña para la frecuencia (0.69), abundancia en función de la densidad $(0.81)$ y abundancia en función de la cobertura (0.93) y considerablemente más alta para la densidad (2.07), cobertura (2.31) y biomasa (3.24). De aquí puede deducirse que, aún dentro de lo correcto de cualquier medida adoptada en la evaluación de la vegetación, las preferencias deben inclinarse sobre todo por la biomasa y mucho menos por cualquier parámetro cuyo espectro de cuantificación esté muy restringido; no debe olvidarse que se está tratando de un ejemplo concreto, y un rango pequeño de variación de la diversidad puede traducirse con facilidad en un alejamiento de las condiciones reales ante débiles errores en el muestreo o ante pequeños cambios en las especies motivados por lạs más variadas causas. Ahora bien, la utilización de la biomasa presenta enormes inconvenientes de tiempo y trabajo invertidos, por lo que cabría preguntarse acerca de la conveniencia de emplear densidad o cobertura; los valores anteriormente citados parecen decantarse en favor de la cobertura, pero aún quedan otras circunstancias por analizar.

Un examen más detallado de la evolución individual de las variaciones para cada caso, demuestra que con predominio de herbáceas apenas si existen diferencias entre densidad y cobertura (incluso las diferencias son poco acusadas para la biomasa); pero la presencia del matorral, que impone grandes coberturas en sí mismo, hace que las trayectorias varíen en favor de la cobertura, que proporciona apreciaciones más claras de los cambios que se producen en la vegetación; por supuesto, la mejor definición del proceso corresponde a la biomasa. En este sentido, cabe anotar que así como el descenso de la línea de densidad es paulatino ante la presencia de leñosas, la cobertura experimenta una fuerte caída inicial (paso de $\mathrm{Mc}$ a $\mathrm{Mm}$ ), mucho más acentuada en el caso de la biomasa. Son las imposiciones a las que da lugar la presencia de individuos de gran tamaño.

En la figura 2 se puede apreciar mejor, para cada uno de los parámetros, la disposición de los diferentes tipos de comunidades por su diversidad y número de especies; la línea gruesa a la derecha de cada gráfica corresponde a la diversidad máxima. 

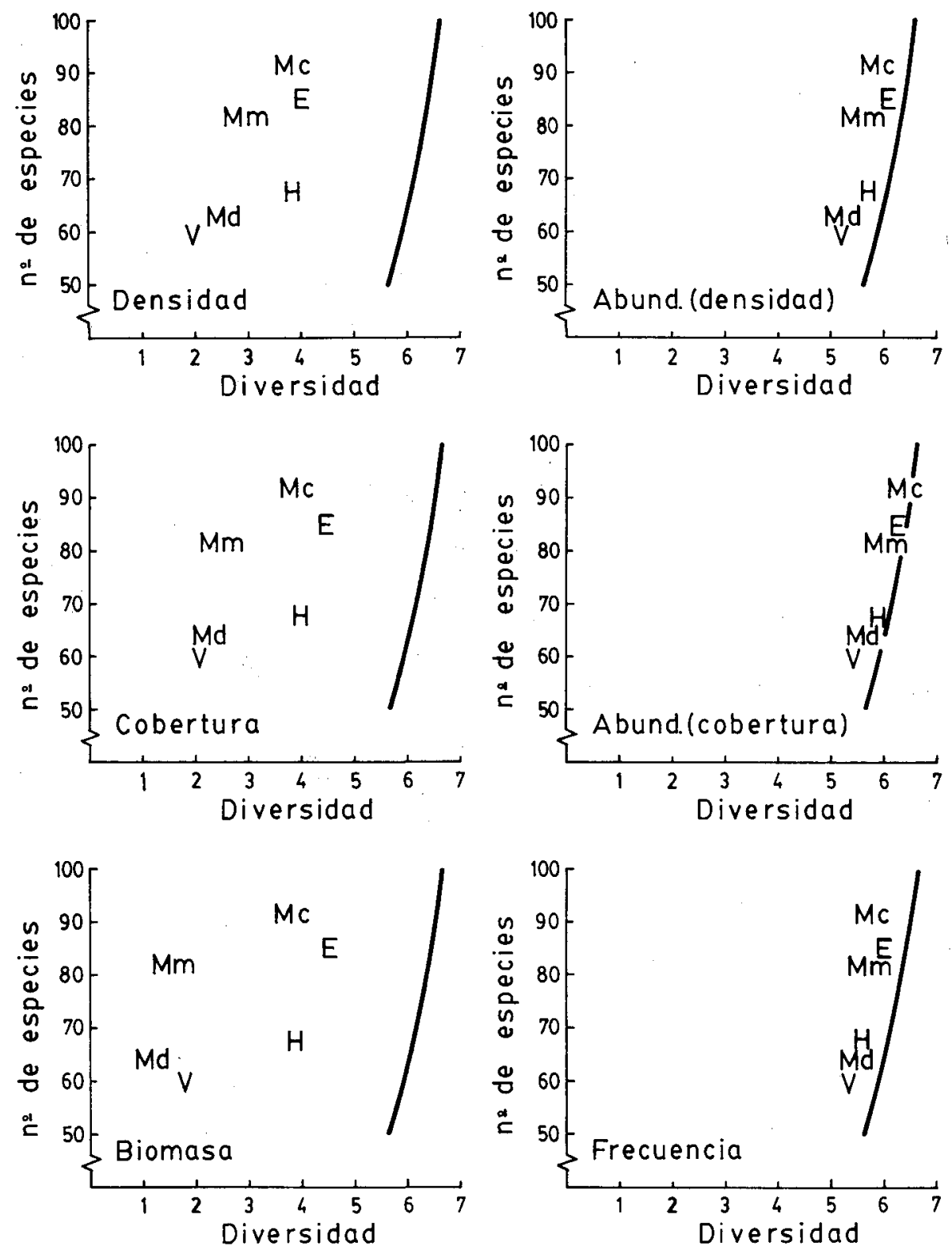

Fig. 2.-Situación de las distintas comunidades tipo al comparar la diversidad media para cada uno de los parámetros de medida con el número medio de especies. La línea gruesa a la derecha de cada gráfica indica la diversidad máxima. 
Como es deducible de la figura anterior, la disposición de las comunidades viene a ser semejante, aunque con amplias fluctuaciones en la separación que aparece entre ellas y respecto a la línea que marca el máximo posible de diversidad. En este sentido, abundancias y frecuencia resultan poco adecuadas, tanto por su ya comentado débil rango de variación como por la proximidad al máximo, lo que es un primer indicio de una alta dependencia respecto al número de especies. Mayor equilibrio se encuentra en densidad, cobertura y biomasa que, en este mismo orden, demuestran ún incremento en el rango de variaciones de la diversidad, particularmente debido al paulatino desplazamiento hacia cifras altas de los pastizales de efímeras $(E)$ y hacia valores bajos del matorral medio (Mm) y denso (Md). Este último hecho ya ha sido suficientemente comentado; en cuanto al primero, cabría resaltar el alto número de individuos pequeños en los pastizales de efímeras, que se iría atenuando al pasar a coberturas y mucho más al considerar la biomasa. Conviene llamar la atención sobre que el mayor número de especies se encuentra en las comunidades más oligotrofas (pastizales de efímeras y matorral con amplios claros), mientras que el menor se relaciona con aquellas de fuerte dominancia, bien sea por especies leñosas (matorral denso) o por herbáceas (vallicares).

Hasta ahora se ha contemplado una parte de la verdad. La otra parte se encuentra en la dependencia que muestra la diversidad respecto a cada una de sus dos componentes; es de esperar que entre la diversidad y sus componentes existan correlaciones altamente significativas, pero como señala HULBERT (1971) respecto a la diversidad y a la riqueza, esto no es una necesidad matemática ni biológica. Con el total de las 30 comunidades muestreadas se obtienen los siguientes coeficientes de correlación:

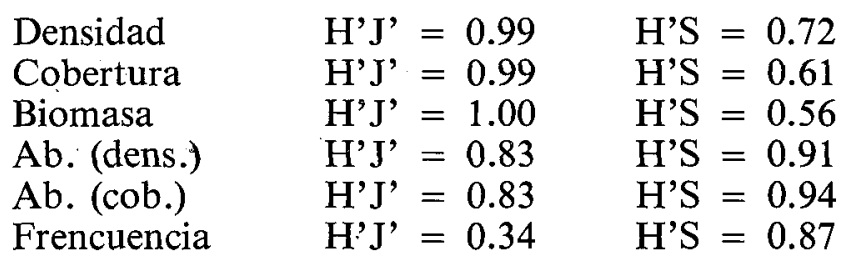

Estos coeficientes son significativamente distintos de cero para una probabilidad del $99 \%$, excepto el de la frecuencia para diversidad y equitabilidad, que no alcanza siquiera la probabilidad del $95 \%$.

En este punto cabe preguntarse si lo que se le pide a un índice de diversidad, dentro de la alta probabilidad de significación, es el equilibrio en la participación de sus componentes o el predominio de una so- 
bre otra. A nuestro entender, la mejor calidad se consigue con un predominio no excesivamente acusado de la equitabilidad, ya que el número de especies es más susceptible a los errores del muestreo. Si se admite esta apreciación, la frecuencia es el peor parámetro a emplear por causas obvias, seguido por la abundancia en función de la cobertura (conviene recordar su acercamiento a los valores máximos de diversidad recogidos en la figura 2) y de la abundancia en función de la densidad. La biomasa, a pesar de la calidad que ha venido demostrando, presenta excesiva dependencia con la equitabilidad en perjuicio de la riqueza (debido a la intervención de especies constituidas por individuos de gran porte), de manera que la decisión puede estar entre densidad y cobertura. Nuestra forma de pensar es que, al menos cuando el muestreo se efectúa sobre especies de porte muy diferente (que es cuando surgen los problemas), debe elegirse la cobertura; ciertamente la densidad demuestra un equilibrio superior, cumpliendo a la vez la premisa del predominio de la equitabilidad, pero la cobertura, por su mayor concordancia con los caracteres fisonómicos, por su superior rango de variación y por su conveniente posición intermedia entre biomasa y densidad, ofrece garantías de no desviar los resultados hacia uno u otro extremo. Una postura menos prudente quizá nos llevaría a preferir la densidad, y posiblemente la elegiríamos de tratarse únicamente de especies herbáceas, aunque sea un valor de importancia bastante más difícil de obtener que la cobertura.

Una última circunstancia puede corroborar nuestra decisión: el establecimiento de todas las correlaciones posibles entre los parámetros empleados. Los coeficientes de correlación, todos ellos significativos para una probabilidad del $99 \%$, se recogen en la figura 3. La cobertura, dentro del grupo preferente densidad-cobertura-biomasa, está fuertemente correlacionada con la densidad y la biomasa, mientras que la densidad baja bastante respecto a la biomasa. La confirmación, en la forma de dendrograma realizado a partir de los mismos coeficientes de correlación (método UPGMA), también queda reflejada en la figura 3. La cobertura muestra una posición intermedia que reúne los caracteres positivos tanto de la biomasa como de la densidad; esta última se dispone a la derecha del grupo prioritario, porque sus coeficientes de correlación indican una unión más fuerte que la de los otros elementos del grupo con aquellos parámetros descartados casi desde un principio, posiblemente por su superior dependencia respecto al número de especies. 


\title{
$D \quad A D \quad C \quad A C \quad F$
}

\author{
$A D \quad 0.91$ \\ C $\quad 0.950 .84$ \\ AC $\quad 0.73 \quad 0.950 .85$ \\ F $\quad 0.610 .860 .750 .92$ \\ B $\quad 0.890 .790 .98 \quad 0.650 .52$
}

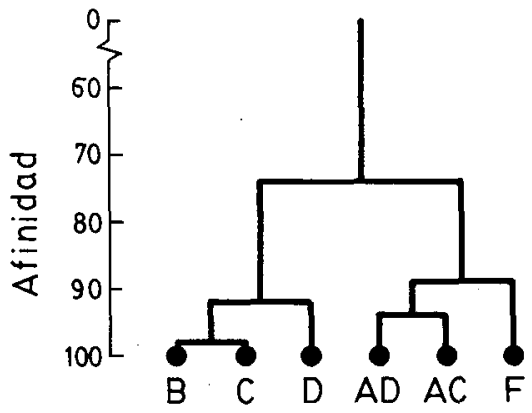

Fig. 3.-Coeficientes de correlación entre todos los pares posibles de parámetros considerados. El dendrograma se ha elaborado a partir de los mismos.

\section{AGRADECIMIENTOS}

Los autores desean expresar su agradecimiento a todos aquellos miembros del Departamento de Ecología y alumnos de esta materia que han colaborado en la costosa tarea de obtener los datos de biomasa. 


\section{BIBLIOGRAFIA}

AUCLAIR, A.N.y F.G. GOFF., (1971). Diversity relations of upland forests in the Western Great Lakes area. Amer. Nat., 105: 499-528.

BAZZAZ, F.A., (1975). Plant especies diversity in old-field successional ecosystems in Southern Illinois. Ecology, 56: 485-488.

CLAVER, I., (1982). Guía para la elaboración de estudios del medio físico: Contenido y metodología. C.E.O.T.M.A. Madrid.

GARCIA DE VICUÑA, B., (1982). Estudio crítico comparativo de varios índices de diversidad. Tesis de Licenciatura. Univ. León.

GOMEZ GUTIERREZ, J.M.; LUIS, E. y A. PUERTO., (1978). El sistema de vaguada como unidad de estudio en pastizales. Rev. Pastos, 8: 219-236.

HULBERT, S.M., (1971). The nonconcept of species diversity: A critique and alternative parameters. Ecology, 52: 557-586.

LOUCKS, O.L., (1970). Evolution of diversity, efficiency, and community stability. Amer. Zool., 10: $17-25$.

LUIS, E. y P. MONSERRAT., (1979). Mapa fitoclimático de la provincia de Salamanca. Estudio integrado y multidisciplinario de la dehesa salmantina. 1. Estudio fisiográfico-descriptivo, 3: $157-181$.

MacINTOSH, R.P., (1967). An index of diversity and the relation of certain concepts to diversity. Ecology, 48: 392-403.

MARGALEF, R., (1974). Ecología. Omega. Barcelona.

MONK, C.D., (1967). Tree species diversity in the Eastern deciduous forest with particular reference to North Central Florida. Amer. Nat., 101: 173-187.

NICHOLSON, S.A. y C.D. MONK, (1974). Plant species diversity in old-field succession on the Georgia Piedmont. Ecology, 55: 1.075-1.085.

ODUM, E.P., (1972). Ecología. Ed. Interamericana. México.

PEET, R.K., (1975). Relative diversity indices. Ecology, 56: 496-498.

PIELOU, E.C., (1975). Ecological diversity. J. Wiley. New York.

PUERTO, A., (1977). Sucesión secundaria en ecosistemas de pastizal. Tesis Doctoral. Univ. Salamanca.

PUERTO, A.; RICO, M. y J.M. GOMEZ GUTIERREZ, (1983). Pautas repetitivas en los pastizales salmantinos. La vaguada como unidad sintética y paisajística. Salamanca. Rev. Provincial de Estudios, 7: 119-144.

RICO, M., (1981). Variabilidad, estructura y composición de pastizales salmantinos. Tesis Doctoral. Univ. Salamanca.

SHAFY, M.I. y G.A. YARRANTON, (1973). Diversity, floristic richness, and species evenness during a secondary (post-fire) succession. Ecology, 54: 897-903.

SHANNON, C.E. y W. WEAVER, (1963). The mathematical theory of communications. Univ. Illinois Press. Urbana. 
STERLING, A.; PECO, B.; CASADO, M.A.; GALIANO, E.F. y F.D. PINEDA, (1984). Influence of microtopography on floristic variation in the ecological succession in grassland. Oikos, 42: 334-342.

TRAMER, E.J., (1975). The regulation of plant species diversity on an early successional oldfield. Ecology, 56: 905-915.

WITTAKER, R.H. y W.A. NIERING, (1975). Vegetation of the Santa Catalina Mountains, Arizona. V. Biomass, production, and diversity along the elevation gradient. Ecology, 56: 77.1-790.

WILLSON, M.F., (1974). Avian community organization and habitat structure. Ecology, 55: 1.017-1.029: 